\title{
Neuronavegação em neurocirurgia
}

\author{
Edmundo Luis Rodrigues Pereira', Daniella Brito Rodrigues², Alzira Leite Gomes², \\ Lorena Oliveira Lima², Maria Luana Carvalho Viegas ${ }^{3}$ \\ Serviço de Neurocirurgia do Hospital Universitário João de Barros Barreto (HUJBB), Belém, PA, Brasil.
}

\section{RESUMO}

A neurocirurgia guiada por imagem permite ao neurocirurgião navegar no interior do crânio, orientado a partir das imagens de tomografia computadorizada (TC) ou ressonância magnética (RM) pré-operatórias, empregando, para isso, sistemas de rastreamento tridimensionais (3D), em tempo real, durante o procedimento cirúrgico. O presente estudo tem como objetivo revisar a literatura acerca das principais aplicações da neuronavegação na neurocirurgia contemporânea. Foi feita uma revisão da literatura por meio de busca na base de dados PubMed, nos últimos 15 anos, nas línguas portuguesa e inglesa, usando os seguintes termos: neuronavegação/neuronavigation, estereotaxia/stereotaxis, glioma surgery, neuroendoscopia/neuroendoscopy, epilepsy surgery, aneurysm. A neuronavegação auxilia a localização espacial, orientando acessos cirúrgicos, o que melhora a qualidade e a segurança do procedimento. Ressalta-se a importância desta nas cirurgias de gliomas em áreas eloquentes, associada a procedimentos neuroendoscópicos e cirurgia de epilepsia e combinada com ultrassom $3 D$ elou RM intraoperatória, com significativa redução da morbidade do ato cirúrgico. O desvio das estruturas após a abertura do crânio e da dura-máter ("brain shift") é considerado o fator limitante para o método, impedindo a perfeita correspondência entre imagens pré-operatórias e a neuronavegação em tempo real, o que pode ser minimizado com a realização de RM intraoperatória (renavegação).

\section{PALAVRAS-CHAVE}

Neuronavegação, epilepsia, neuroendoscopia, doenças da coluna vertebral, glioma, neuroimagem, aneurisma intracraniano.

\section{ABSTRACT}

Neuronavigation in neurosurgery

The image-guided neurosurgery allows the neurosurgeon to navigate within patient's skull, using preoperative images as a guide, through the use of $3 D$ tracking systems, during the surgical procedure. This study aims to review the literature on the main applications of neuronavigation in modern neurosurgery. We performed a literature search through the database PubMed in the last 15 years in Portuguese and English, using the following terms: neuronavegação/neuronavigation, estereotaxia/stereotaxis, glioma surgery, neuroendoscopia/neuroendoscopy, epilepsy surgery, aneurysm. Neuronavigation improves the efficacy and security of surgery, with emphasis in surgery of gliomas in or around eloquent areas, association with neuroendoscopy and epilepsy surgery, as well combined with $3 D$ ultrasound and intraoperative MRI. Dislocation of brain and structures after opening cranial vault and dura ("brain shift") still is a limitation to the perfect correspondence between the surgery and preoperative images of neuronavigation, which can be solved with intraoperative MRI.

\section{KEYWORDS}

Neuronavegation, epilepsy, neuroendoscopy, spinal diseases, glioma, neuroimaging, intracranial aneurysm.

1 Neurocirurgião do Hospital Universitário João de Barros Barreto (HUJBB), professor de Neurologia da Universidade Federal do Pará (UFPA), Belém, PA, Brasil.

2 Acadêmicas de Medicina da Universidade do Estado do Pará (UEPA), Belém, PA, Brasil.

3 Acadêmica de Medicina da UFPA, Belém, PA, Brasil. 


\section{Introdução}

A neuronavegação emana do desenvolvimento da neurorradiologia, sendo decorrente da introdução e da integração de altas tecnologias aplicadas à moderna neurocirurgia. ${ }^{1}$ A neurocirurgia guiada por imagem permite ao neurocirurgião se orientar no interior do crânio, "navegando" em tempo real no interior do cérebro usando como referencial imagens pré-operatórias alinhadas a sistemas de rastreamento $3 \mathrm{D}$, durante o procedimento cirúrgico. A partir de um procedimento simples de calibração dos instrumentos a serem utilizados na cirurgia, a posição tridimensional de determinado instrumento no interior do crânio pode ser transmitida a uma estação de informática que correlaciona a posição do instrumento com as imagens pré-operatórias de tomografia computadorizada (TC) ou ressonância magnética (RM), indicando ao cirurgião, em tempo real, o trajeto ou o caminho a ser seguido e a localização de uma lesão no interior do cérebro. ${ }^{2-4}$

Esse método acrescentou significativa evolução na neurocirurgia, e a aplicação desse sistema na neurocirurgia contemporânea é praticamente ilimitada, pois fornece maior segurança ao cirurgião e melhor resultado para o paciente. ${ }^{4-7} \mathrm{O}$ presente estudo tem como objetivo analisar as principais aplicações da neuronavegação na neurocirurgia comtemporânea, destacando suas principais indicações, desvantagens e perspectivas quanto às novas tecnologias associadas.

Foi realizada uma revisão da literatura por meio de busca na base de dados PubMed nos últimos 15 anos nas línguas portuguesa e inglesa, usando os seguintes termos: neuronavegação/neuronavigation, estereotaxia/ stereotaxis, glioma surgery, neuroendoscopia/neuroendoscopy, epilepsy surgery, aneurysm. Os trabalhos foram avaliados quanto a resultados, relevância clínica, desenho do estudo e nível de evidência.

\section{Neuronavegação e neuroendoscopia}

Para Jaimovich et al., ${ }^{8}$ as principais indicações da neuronavegação na neuroendoscopia são: cirurgias para hidrocefalia, anatomia distorcida e forame de Monro pequeno, assim como líquido cefalorraquidiano (LCR) turvo ou hemorrágico; nas cirurgias para cisto aracnoide, é útil para localizar o ponto de entrada em cistos temporais, comunicar cistos suprasselares, da lâmina quadrigêmea e ependimários a ventrículos pequenos e em cistos de septo pelúcido sintomáticos; para biópsia ou ressecção de tumores cerebrais e comunicação de cistos ventriculares; para cirurgia de epilepsia, permite o implante de eletrodos no corno temporal do ventrículo lateral, a calosotomia e a desconexão de hamartomas hipotalâmicos. Esses autores analisaram 14 pacientes operados com a combinação entre as duas tecnologias, com precisão sempre inferior a $1 \mathrm{~mm}$, e concluíram que essas são técnicas seguras e eficazes, quando a patologia é passível de resolução via endoscópica e quando os ventrículos são pequenos. Concluíram também que os índices de complicações com essa combinação são extremamente baixos, o que corrobora com a literatura, entretanto, outros autores destacam a dificuldade de acesso a ventrículos pequenos quando a precisão não é inferior a $1 \mathrm{~mm} .{ }^{9,10}$

Maeda et al. ${ }^{11}$ relataram a fenestração endoscópica de cisto ventricular multiloculado guiada por neuronavegação e concluíram que a falta de referências anatômicas bem definidas assim como as inúmeras septações são fatores que dificultam o manejo do endoscópio dentro do sistema ventricular. Portanto, esses autores avaliaram que a neuronavegação torna o procedimento endoscópico mais seguro e com eficácia maior.

Carvi Nievas et al. ${ }^{12}$ relataram a técnica de implantação de um cateter transseptal e biventricular com derivação peritoneal distal em pacientes com hidrocefalia pós-hemorrágica e acentuada dilatação dos ventrículos laterais assistida por neuronavegação. Esses autores, após realizarem essa técnica com neuronavegação em 11 pacientes, não tiveram complicações e não houve necessidade de revisão de nenhum sistema. Concluíram que essa é uma opção válida para o tratamento de hidrocefalia pós-hemorragia, pois reduz o número de catéteres e minimiza o tempo do procedimento cirúrgico, assim como os riscos de infecção.

Hermann et al. ${ }^{13}$ avaliaram a cirurgia, guiada por neuronavegador, de 26 crianças com hidrocefalia e anatomia ventricular distorcida. Nesse estudo, os autores demonstram as vantagens da utilização dessa tecnologia para a colocação de shunt em crianças. Não houve necessidade de punções ventriculares repetidas, tampouco complicações durante as cirurgias. No pós-operatório, todas as crianças tiveram adequado posicionamento do shunt, no entanto, esses autores tiveram três casos de infecções tardias e uma revisão de válvula. Apesar disso, consideraram que o sistema de neuronavegação permite a colocação do cateter de forma segura, especialmente em crianças e recém-nascidos prematuros, reduzindo a necessidade de tentativas de repetidas punções ventriculares.

\section{Cirurgia de epilepsia}

A neuronavegação é importante na cirurgia de epilepsia porque fornece orientação anatômica para o 
direcionamento de ablações das áreas epileptogênicas (por exemplo, amigdaloipocampectomia), implantação de eletrodos profundos e colocação das placas de registro subdural, otimizando a estimulação cortical e, na localização intraoperatória de áreas eloquentes, tomografia por emissão de pósitrons (PET) e tomografia computadorizada por emissão de fóton único (SPECT).$^{14}$

Chamoun et al. ${ }^{15}$ destacam que a localização exata da zona epileptogênica é de suma importância na cirurgia da epilepsia, e a ampla utilização da neuronavegação melhora a precisão na colocação das placas subdurais. Os autores descrevem duas técnicas que ampliam a utilidade de neuronavegação em doentes com epilepsia submetidos à monitorização com eletrodos subdurais e que não exigem dispositivos adicionais, software ou conhecimentos mais sofisticados de informática. Esses autores consideraram seus resultados satisfatórios e, portanto, destacam a utilidade da colocação precisa dessas placas com o uso do neuronavegador. Esse estudo invasivo com a implantação de placas subdurais é de extrema utilidade em casos selecionados de pacientes candidatos à cirurgia de epilepsia. Os eletrodos permitem não só o registro do início e a propagação das crises, como também mapear as áreas eloquentes do cérebro do paciente. Tais pontos no córtex do paciente podem ser armazenados no neuronavegador para servirem durante a cirurgia para guiar a ressecção cirúrgica após a retirada das placas. O mapeamento funcional fica armazenado no neuronavegador, podendo servir como importante referência para futuras reoperações que porventura se tornem necessárias.

Wellmer et al. ${ }^{16}$ analisaram a utilidade da neuronavegação aliada à RM intraoperatória morfométrica nas cirurgias para displasias corticais focais e consideraram essa combinação útil e eficaz, porque a análise dos dados funcionais e anatômicos facilita, principalmente, a ressecção de displasias pequenas e profundas. Stone e Rutka ${ }^{14}$ destacaram a importância da adequada orientação anatômica; a utilidade do neuronavegador para a realização de cirurgias de epilepsia seguras e eficazes pode, por exemplo, auxiliar no planejamento da posição do flap ósseo para maximizar o acesso seguro durante abordagens inter-hemisféricas para calosotomias, para selecionar o ângulo das abordagens transtemporais na lobectomia temporal, na realização das amigdaloipocampectomias seletivas. ${ }^{17-19} \mathrm{Em}$ uma série retrospectiva de 217 pacientes que foram submetidos à colocação de eletrodos guiada por neuronavegação, o procedimento permitiu adequado planejamento cirúrgico em $96 \%$ dos casos. ${ }^{20}$

Schwartz et al. ${ }^{21}$ propuseram que a RM intraoperatória pode padronizar a ressecção nas amigdaloipocampectomias radicais, e essa abordagem pode reduzir a variabilidade dos resultados cirúrgicos, entretanto, mais estudos são necessários para estabelecer os benefícios da melhor orientação anatômica intraoperatória com ressonância magnética em cirurgia de epilepsia, especialmente à luz de seu custo considerável. As ressecções seletivas do lobo temporal, das estruturas mesiais, preservando-se o neocórtex, são tecnicamente facilitadas pela neuronavegação, que possibilita uma abordagem rápida e direta dessas estruturas, com incisões e craniotomias menores.

\section{Coluna}

A neuronavegação na cirurgia espinhal é considerada eficaz e precisa, principalmente nas instrumentações complexas da coluna toracolombar. ${ }^{22}$ Essa tecnologia melhora a precisão da instrumentação e diminui o risco de lesão às estruturas neurovasculares adjacentes. Além disso, a neuronavegação evita a exposição à radiação, presente nos procedimentos tradicionais que utilizam fluoroscopia. Essa técnica permite feedback em tempo real para o cirurgião da instrumentação nos planos coronal, sagital e axial, possibilitando que quaisquer erros na trajetória, no tamanho do parafuso, ou possível lesão a estruturas adjacentes, possam ser corrigidos. ${ }^{22-25}$

Estudo de metanálise comparando a precisão na colocação do parafuso transpedicular na coluna toracolombar sem navegação e com navegação encontrou uma precisão média de $86,6 \%$ e de $93,7 \%$, respectivamente. ${ }^{26}$ Na série de Tormenti et al. ${ }^{22}$, toda a instrumentação lombar/lombossacral foi realizada com precisão dentro do pedículo em $100 \%$ dos casos, entretanto, a análise da instrumentação torácica mostrou que $2,4 \%$ dos parafusos ( $1,2 \%$ de todos os parafusos colocados) violaram o pedículo. Nesse estudo, não houve invasão de estruturas neurovasculares nem alteração significativa no diâmetro do canal espinal e considerou-se, portanto, a orientação da imagem guiada por neuronavegação superior à tradicional fluoroscopia.

Para Bescós et al. ${ }^{24}$ a estabilização do segmento C1C2 evoluiu com o aparecimento de várias técnicas de fixação sublaminares, transarticular ou interarticular e, especialmente, com a introdução de sistemas de neuronavegação. Esses autores consideraram que a fixação de C1-C2 com o auxílio da neuronavegação é segura e proporciona uma taxa elevada de bons resultados, com poucas complicações. No estudo de Tormenti et al., ${ }^{22}$ em uma análise da instrumentação de um total de 164 parafusos transpediculares na coluna toracolombar, encontrou-se violação do pedículo em apenas dois parafusos $(1,2 \%)$, significativamente superior à taxa de $5,2 \%$ no grupo de pacientes operados com fluoroscopia. Esses autores concluíram que a orientação por neuronavegação possui uma precisão (aumento de $98,22 \%$ ) 
que supera as taxas relatadas com outros sistemas de orientação de imagem, como a fluoroscopia virtual.

\section{Tumores}

A neuronavegação ajuda a reduzir a capacidade de invasão, melhora a qualidade da cirurgia e reduz o tempo de operação. A neuronavegação funcional combinada com ultrassom $3 \mathrm{D}$ e ressonância magnética intraoperatória permite, na maioria dos pacientes, a ressecção de lesões profundamente localizadas no cérebro. Seu uso é consagrado nas cirurgias para ressecção de tumores cerebrais, especialmente os gliomas, considerando que maior citorredução tumoral e preservação de áreas eloquentes são obtidas nas cirurgias com neuronavegador. ${ }^{27}$ Pode ser utilizada desde a localização da craniotomia até a localização de fibras da substância branca, áreas eloquentes e estruturas neurovasculares durante a ressecção de neoplasias intra-axiais. ${ }^{28}$

Musolino, ${ }^{29}$ ao analisar as novas estratégias de tratamento cirúrgico para tumores hipofisários, considerou que esse método guia o cirurgião, desde a abordagem do assoalho da sela até a localização dos instrumentos em relação ao tumor e às estruturas adjacentes, como as carótidas e os nervos ópticos. Esse autor também considera a associação ao endoscópio ou à ressonância intraoperatória e destaca sua utilização em reabordagens, quando a perda das estruturas ósseas pode dificultar o acesso da linha média através do endoscópio. Silva et al., ${ }^{28}$ após análise do uso da neuronavegação nas ressecções dos meningiomas, concluíram que os principais objetivos de neuronavegação nesses procedimentos estão em otimizar as craniotomias, diminuir as taxas de danos aos seios da dura-máter e promover uma ressecção mais segura do tumor.

Para Sun et al. ${ }^{27}$ a ressonância magnética intraoperatória combinada com a neuronavegação é um método seguro e eficaz para ressecções de tumores nas radiações ópticas, preservando o campo visual do paciente. A combinação entre as duas técnicas permitiu diminuição na taxa residual na ressecção dos gliomas $(5,3 \%)$ e não houve tumor residual entre os não gliomas. Nesse estudo, o campo visual após a cirurgia melhorou em cinco casos (11,4\%), não houve nenhuma alteração em 36 casos $(81,8 \%)$ e em apenas três casos houve piora do déficit neurológico $(6,8 \%)$. Outros autores consideram também o uso combinado de tratografia, ressonância magnética funcional e neuronavegação como ferramentas seguras nas ressecções de gliomas de alto grau. ${ }^{30}$

Hwang e $\mathrm{Ho}^{31}$ destacaram o uso da neuronavegação nas ressecções de tumores clivais, considerada extensa, traumática e invasiva. Esse estudo não obteve complicações e houve redução do tempo de internação. Desse modo, a abordagem transesfenoidal guiada por neuronavegador foi considerada uma alternativa viável e minimamente invasiva para o tratamento cirúrgico de tumores clivais.

\section{Vascular}

Apesar dos avanços na tecnologia dos sistemas de neuronavegação, a aplicação dessas tecnologias no campo da neurocirurgia vascular é restrita devido às limitações em resolução espacial e suscetibilidade maior dessas modalidades de imagem para artefatos como pinos de fixação, clipes de aneurismas e materiais embólicos previamente implantados. No entanto, muitas dessas limitações podem ser minimizadas com o uso da angiografia digital. ${ }^{32}$

Kim et al..$^{33}$ analisaram o manejo de aneurismas da porção distal da artéria cerebral anterior. Nesse estudo, não houve complicações cirúrgicas e o sistema de neuronavegação permitiu a identificação dos aneurismas com elevada precisão (média de $0,88 \mathrm{~mm}$ ) em todos os pacientes analisados. Consideraram, desse modo, que a neuronavegação ofereceu significativos benefícios adicionais no que concerne a menor craniotomia e precisão intraoperatória, tornando a cirurgia minimamente invasiva. Outro estudo analisou os resultados cirúrgicos de 16 pacientes com aneurismas da circulação anterior operados com auxílio do neuronavegador. Neste, a avaliação intraoperatória permitiu uma craniotomia mais exata e precisa em relação, principalmente, aos aneurismas da artéria cerebral média, e a apresentação em 3D dos aneurismas foi crucial para a identificação e a dissecção dos aneurismas. ${ }^{34}$

Rohde et ll. $^{35}$, ao analisarem a utilidade da neuronavegação em tumores e lesões vasculares tratados por meio de abordagens pela base do crânio, concluíram que os sistemas de neuronavegação que exibem as reconstruções 3D da lesão, assim como dos vasos, nervos e tratos adjacentes durante a cirurgia, são ferramentas importantes na gestão neurocirúrgica de lesões da base do crânio. Em sua casuística de cavernomas do tronco cerebral, o neuronavegador foi considerado útil para a definição da melhor abordagem cirúrgica em relação ao trato piramidal e aos núcleos do tronco cerebral.

Dzierzanowski et al. ${ }^{36}$ destacaram que os sistemas de neuronavegação também podem ser utilizados em estudos morfométricos, e as vantagens desse método são o número praticamente ilimitado de resultados que podem ser analisados em detalhes e a possível repetibilidade da técnica. Para Mathiesen et al., ${ }^{37}$ a análise do uso da neuronavegação nas cirurgias para malformações arteriovenosas permitiu destacar que a navegação amenizou a complexidade intraoperatória dos vasos patológicos da malformação arteriovenosa (MAV), porque o contorno tridimensional da vasculatura (ir- 
rigação, nidus e veias de drenagem) forneceu um meio para adaptar as estratégias de ressecção, definir os planos de dissecação e interpretar achados intraoperatórios, o que melhorou os resultados cirúrgicos.

\section{Desvio cerebral após craniotomia ("brain-shift")}

A neuronavegação é baseada em imagens pré-operatórias, sendo, portanto, sujeita a um erro que às vezes pode inviabilizar ou diminuir a eficácia do procedimento. Quando o crânio e a dura-máter são abertos, tanto a forma como o posição do cérebro podem mudar devido à alteração do ambiente físico dentro do crânio, com saída de liquor, entrada de ar e outras modificações regionais no formato dos lobos cerebrais. ${ }^{1,38,39} \mathrm{~A}$ abertura do sistema ventricular com drenagem liquórica e a ressecção da massa intracerebral resultam em deformação ainda maior do cérebro. ${ }^{40-42}$ Isso se deve ao fato de a aquisição da imagem ter sido realizada com o encéfalo estático, o qual permanece imóvel até a abertura do crânio e da dura-máter, momento em que ocorre deslocamento das estruturas intracranianas, situação a partir da qual o cirurgião pode ser induzido ao erro, pois está sendo guiado por uma imagem anterior, que já não corresponde à realidade. Essa modificação no posicionamento do encéfalo durante a cirurgia é conhecida como "brain shift", significando, literalmente, "desvio cerebral". 138,39

Diversos estudos têm se dedicado a análise e classificação dos erros em neuronavegação, especialmente o "brain-shift" ${ }^{38}$ No estudo de Dorward et al. ${ }^{41}$, a deformação média foi de $4,6 \mathrm{~mm}$ na superfície cortical após a abertura da dura-máter e de 5,1 mm após ressecção tumoral, enquanto para Hill et al. ${ }^{43}$ a deformação da superfície cerebral era de 4,4 mm logo após a abertura da dura-máter e de 5,6 mm cerca de 1 hora depois. Nimsky et al. ${ }^{40}$ classificaram a deformação em três níveis: alta (7,0 mm), moderada (3,0-6,9 mm) e baixa (0-2,9 mm).

Apesar disso, a magnitude e o impacto do "brain-shift" ainda são pouco compreendidos, e as causas de distorção do cérebro são tanto fisiológicas e terapêuticas quanto decorrentes de alterações físicas. A administração de medicamentos diuréticos e ventilação mecânica é considerada fator terapêutico. O posicionamento do paciente, a retirada do líquido cefalorraquidiano, a compressão ventricular, a retração e a remoção do tecido tumoral são considerados fatores físicos que, em ambos os casos, alteram a conformação da estrutura dos hemisférios e lobos cerebrais. ${ }^{41}$

A deformação do cérebro leva a significativo erro de interpretação e aplicação do sistema de neuronavegação, e muitos estudos têm sido feitos para lidar com essa limitação, que incluem realizar exame de RM intraoperatória, com o intuito de atualizar as imagens pré-operatórias e calibrar novamente o sistema de recepção óptica a partir das novas imagens obtidas após a craniotomia. Outra tentativa de solucionar o "brain shift" tem sido a utilização de "software" usando a imagem em tempo real do ultrassom para corrigir as imagens de ressonância magnética pré-operatória. ${ }^{38,44}$

\section{Perspectivas}

A sobreposição de dados de RM funcional, assim como de RM com perfusão e difusão, permite a orientação ao neurocirurgião com o mínimo possível de margem de erro na localização, possibilitando a reavaliação da lesão e tecido circundante durante $o$ ato operatório. Modalidades de imagem que usam parâmetros com base nas características metabólicas do tecido neural, tais como ressonância magnética funcional (RMf), angio-RM, RM com espectroscopia, são aplicações que refinam a neuronavegação e abrangem toda a monitorização do paciente no intraoperatório. O SPECT e o PET têm sido considerados mais eficazes quando associados com neuronavegador. A neuronavegação combinada com ultrassonografia intraoperatória tem sido considerada ferramenta decisiva e eficaz, devido ao baixo custo e à relativa simplicidade e tolerabilidade do método. A ultrassonografia fornece imagens em tempo real sem uma carga de radiação para o paciente e não exige quaisquer requisitos especiais no arsenal neurocirúrgico ou para o ambiente da sala de operações. ${ }^{1}$ Alguns autores consideram que, em um futuro próximo, os robôs provavelmente serão integrados com neuronavegação e a telerrobótica pode permitir o desempenho de procedimentos neurocirúrgicos a distância, em localizações até mesmo remotas. ${ }^{1,45,46}$

\section{Considerações finais}

A neuronavegação modificou a neurocirurgia contemporânea, sendo particularmente útil nas lesões cerebrais pequenas, profundas e em áreas eloquentes. A associação da neuronavegação com RM intraoperatória e ultrassom 3D proporciona uma correspondência mais fidedigna entre as imagens pré-operatórias e o ato cirúrgico. Contudo, o "brain shift" ainda é considerado uma limitação à perfeita correspondência entre o ato cirúrgico e as imagens pré-operatórias da neuronavegação, problema que pode ser resolvido com o emprego de RM intraoperatória e ultrassom 3D. 


\section{Referências}

1. Enchev Y. Neuronavigation: geneology, reality, and prospects. Neurosurg Focus. 2009;27(3):E11.

2. Enchev YP, Popov RV, Romansky KV, Marinov MB, Bussarsky VA. Cranial neuronavigation--a step forward or a step aside in modern neurosurgery. Folia Med (Plovdiv). 2008;50(2):5-10.

3. Enchev YP, Popov RV, Romansky KV, Marinov MB, Bussarsky VA. Effect of the type of image study (CT or MRI) on some parameters of neuronavigation-assisted procedures. Folia Med (Plovdiv). 2008;50(3):47-52.

4. Enchev YP, Popov RV, Romansky KV, Marinov MB, Bussarsky VA. Neuronavigated surgery of intracranial cavernomas--enthusiasm for high technologies or a gold standard? Folia Med (Plovdiv). 2008;50(2):11-7.

5. Enchev YP, Popov RV, Romansky KV, Marinov MB, Bussarsky VA. Role of neuronavigation in the resection of intracranial arteriovenous malformations. Folia Med (Plovdiv). 2008;50(1):40-5.

6. Enchev Y, Bozinov O, Miller D, Tirakotai W, Heinze S, Benes $\mathrm{L}$, et al. Image-guided ultrasonography for recurrent cystic gliomas. Acta Neurochir (Wien). 2006;148(10):1053-63.

7. Enchev Y, Oi S. Historical trends of neuroendoscopic surgical techniques in the treatment of hydrocephalus. Neurosurg Rev. 2008;31(3):249-62.

8. Jaimovich R, Sosa F, Cuccia V, Zuccaro G. Neuroendoscopia guiada por neuronavegación. Rev Argent Neurocir. 2007;21(1)22-99.

9. Nonaka Y, Oi S, Samii A, Paterno V, Feigl GC, Lüdemann $W$, et al. Neuronavigational neuroendoscopic surgery. Frameless free-hand maneuvering of a handy rigidrod neuroendoscope on visualized three-dimensional computerized image guidance: trajectory to the prepontine cistern in cadaver study. Childs Nerv Syst. 2006;22(1):1827.

10. Kim IY, Jung S, Moon KS, Jung TY, Kang SS. Neuronavigation-guided endoscopic surgery for pineal tumors with hydrocephalus. Minim Invasive Neurosurg. 2004;47(6):365-8.

11. Maeda AK, Aguiar LR, Francisco NA, Cavalheiro S, Zymberg ST, Carboni Junior $P$, et al. Fenestração endoscópica de cisto ventricular multiloculado guiada por neuronavegação. J Bras Neurocirurg. 2003;14(3):115-9.

12. Carvi Nievas MN. Neuronavigation-assisted single transseptal catheter implantation and shunt in patients with posthemorrhagic hydrocephalus and accentuated lateral ventricles dilatation. Surg Neurol Int. 2011;2:34.

13. Hermann EJ, Capelle HH, Tschan CA, Krauss JK. Electromagnetic-guided neuronavigation for safe placement of intraventricular catheters in pediatric neurosurgery. $J$ Neurosurg Pediatr. 2012;10(4):327-33.

14. Stone SS, Rutka JT. Utility of neuronavigation and neuromonitoring in epilepsy surgery. Neurosurg Focus. 2008;25(3):E17.

15. Chamoun RB, Nayar VV, Yoshor D. Neuronavigation applied to epilepsy monitoring with subdural electrodes. Neurosurg Focus. 2008;25(3):E21.

16. Wellmer J, Parpaley Y, von Lehe M, Huppertz HJ. Integrating magnetic resonance imaging postprocessing results into neuronavigation for electrode implantation and resection of subtle focal cortical dysplasia in previously cryptogenic epilepsy. Neurosurgery. 2010;66(1):187-94.

17. Hodaie M, Musharbash A, Otsubo H, Snead OC 3rd, Chitoku $S$, Ochi $A$, et al. Image-guided, frameless stereotactic sectioning of the corpus callosum in children with intractable epilepsy. Pediatr Neurosurg. 2001;34(6):286-94.

18. Murphy MA, O'Brien TJ, Cook MJ. Insertion of depth electrodes with or without subdural grids using frameless stereotactic guidance systems--technique and outcome. Br J Neurosurg. 2002;16(2):119-25.

19. Wurm G, Wies W, Schnizer M, Trenkler J, Holl K. Advanced surgical approach for selective amygdalohippocampectomy through neuronavigation. Neurosurgery. 2000;46(6):137782.

20. De Almeida AN, Olivier A, Quesney F, Dubeau F, Savard G, Andermann F. Efficacy of and morbidity associated with stereoelectroencephalography using computerized tomography--or magnetic resonance imaging-guided electrode implantation. J Neurosurg. 2006;104(4):483-7.

21. Schwartz TH, Marks D, PakJ, Hill J, Mandelbaum DE, Holodny $\mathrm{Al}$, et al. Standardization of amygdalohippocampectomy with intraoperative magnetic resonance imaging: preliminary experience. Epilepsia. 2002;43(4):430-6.

22. Tormenti MJ, Kostov DB, Gardner PA, Kanter AS, Spiro RM, Okonkwo DO. Intraoperative computed tomography image-guided navigation for posterior thoracolumbar spinal instrumentation in spinal deformity surgery. Neurosurg Focus. 2010;28(3):E11.

23. Rath SA, Moszko S, Schäffner PM, Cantone G, Braun $\mathrm{V}$, Richter HP, et al. Accuracy of pedicle screw insertion in the cervical spine for internal fixation using frameless stereotactic guidance. J Neurosurg Spine. 2008;8(3):23745.

24. Bescós A, Muñoz J, Colet S, Domínguez CJ, Cardiel I, Florensa R. [Posterior C1-C2 arthrodesis. Experience in transarticular and interarticular fixation in 36 patients]. Neurocirugia (Astur). 2011;22(2):140-9.

25. Thorat JD, Rajendra T, Thirugnanam A, Ng IH. Singlestage posterior midline approach for dumbbell tumors of the thoracic spine, with intraoperative CT guidance. Surg Neurol Int. 2011;2:31.

26. Kosmopoulos V, Schizas C. Pedicle screw placement accuracy: a meta-analysis. Spine (Phila Pa 1976). 2007;32(3):E111-20.

27. Sun GC, Chen XL, Zhao Y, Wang F, Hou BK, Wang YB, et al. Intraoperative high-field magnetic resonance imaging combined with fiber tract neuronavigation-guided resection of cerebral lesions involving optic radiation. Neurosurgery. 2011;69(5):1070-84.

28. Silva DO, Matis GK, Costa LF, Kitamura MA, Birbilis TA, Azevedo Filho HR. Intraventricular trigonal meningioma: Neuronavigation? No, thanks! Surg Neurol Int. 2011;2:113.

29. Musolino NRC. Tumores hipofisários: opções terapêuticas atuais e perspectivas futuras de tratamento. Arq Bras Endocrinol Metab. 2003;47(4):482-91.

30. Prat R, Inmaculada G, Almudena L, Gaspar R, Romá AR. Impact of the combined use of tractography, functional magnetic resonance imaging and brain neuronavigation on high grade glioma extent of resection. Rev Chil Neurocir. 2011;36(1):40-5.

31. Hwang PY, Ho CL. Neuronavigation using an image-guided endoscopic transnasal-sphenoethmoidal approach to clival chordomas. Neurosurgery. 2007;61(Suppl 2):212-7.

32. Leng LZ, Rubin DG, Patsalides A, Riina HA. Fusion of intraoperative three-dimensional rotational angiography and flat-panel detector computed tomography for cerebrovascular neuronavigation. World Neurosurg. 2013;79(3-4):504-9.

33. Kim TS, Joo SP, Lee JK, Jung S, Kim JH, Kim SH, et al. Neuronavigation-assisted surgery for distal anterior 
cerebral artery aneurysm. Minim Invasive Neurosurg. 2007;50(3):140-4.

34. Schmid-Elsaesser R, Muacevic A, Holtmannspötter M, Uhl E, Steiger HJ. Neuronavigation based on CT angiography for surgery of intracranial aneurysms: primary experience with unruptured aneurysms. Minim Invasive Neurosurg. 2003;46(5):269-77.

35. Rohde V, Spangenberg P, Mayfrank L, Reinges M, Gilsbach JM, Coenen VA. Advanced neuronavigation in skull base tumors and vascular lesions. Minim Invasive Neurosurg. 2005;48(1):13-8.

36. Dzierzanowski J, Słoniewski P, Rut M. Morphometry of the pterional and pterional-orbitozygomatic approaches to the basilar artery bifurcation by the use of neuronavigation systems: a new technical concept. Folia Morphol (Warsz). 2008;67(4):267-72.

37. Mathiesen T, Peredo I, Edner G, Kihlström L, Svensson $\mathrm{M}$, Ulfarsson $\mathrm{E}$, et al. Neuronavigation for arteriovenous malformation surgery by intraoperative three-dimensional ultrasound angiography. Neurosurgery. 2007;60(Suppl 2):345-50.

38. Wang MN, Song ZJ. Classification and analysis of the errors in neuronavigation. Neurosurgery. 2011;68(4):1131-43.

39. Dorward NL, Alberti O, Velani B, Gerritsen FA, Harkness WFJ, Kitchen ND, et al. Postimaging brain distortion: magnitude, correlates, and impact on Neuronavigation. Neurosurg Focus. 1999;6(3):E6.

40. Nimsky C, Ganslandt O, Cerny S, Hastreiter P, Greiner G, Fahlbusch R. Quantification of, visualization of, and compensation for brain shift using intraoperative magnetic resonance imaging. Neurosurgery. 2000;47(5):1070-9.

41. Dorward NL, Alberti O, Velani B, Gerritsen FA, Harkness WF, Kitchen ND, et al. Postimaging brain distortion: magnitude, correlates, and impact on neuronavigation. $J$ Neurosurg. 1998;88(4):656-62.

42. Hartkens T, Hill DL, Castellano-Smith AD, Hawkes DJ, Maurer CR Jr, Martin AJ, et al. Measurement and analysis of brain deformation during neurosurgery. IEEE Trans Med Imaging. 2003;22(1):82-92.

43. Hill DL, Maurer CR Jr, Maciunas RJ, Barwise JA, Fitzpatrick JM, Wang MY. Measurement of intraoperative brain surface deformation under a craniotomy. Neurosurgery. 1998;43(3):514-26.

44. Roberts DW, Hartov A, Kennedy FE, Miga MI, Paulsen KD. Intraoperative brain shift and deformation: a quantitative analysis of cortical displacement in 28 cases. Neurosurgery. 1998;43(4):749-58.

45. Foroglou N, Zamani A, Black P. Intra-operative MRI (iop-MR) for brain tumour surgery. Br J Neurosurg. 2009;23(1):14-22.

46. Ma B, Ellis RE. Unified point selection and surface-based registration using a particle filter. In: Duncan JS, Gerig G, editors. Medical Image Computing and Computer-Assisted Intervention (MICCAI 2005). Berlin: Springer; 2005. p. 75-82.

Endereço para correspondência

Edmundo Luis Rodrigues Pereira

Rua dos Pariquis, 1838, ap. 802, Jurunas

66033-590 - Belém, PA, Brasil

E-mail: eluis@ufpa.br 